\title{
Persistent idiopathic facial pain
}

INSERM

\section{Source}

INSERM. (1999). Orphanet: an online rare disease and orphan drug data base. Persistent idiopathic facial pain. ORPHA:398147

A rare neurological disease characterized by a generally deep, poorly localized, persistent facial pain that does not present characteristics of a cranial neuralgia and which cannot be attributed to another disorder. 